\title{
Management of Stoma Prolapse: A Novel Technique for Repair
}

\author{
Roger Christopher Gill*1, Syed Omair Nadeem ${ }^{2}$ and Tabish Umer Chawla ${ }^{3}$ \\ ${ }^{1}$ Department of Surgery, the Aga Khan University Hospital, Pakistan \\ ${ }^{2}$ Medical Student, the Aga Khan University Hospital Medical College, Pakistan \\ ${ }^{3}$ Section Head General Surgery, the Aga Khan University Hospital, Pakistan
}

Received: March 01,2018; Published: March 14, 2018

*Corresponding author: Roger Christopher Gill, Fellow Trauma \& General Surgery, Department of Surgery, The Aga Khan University Hospital, Karachi, Pakistan, Tel: 923009234726; Email: christo214@gmail.com

Abstract

We present to you a case of stoma prolapse in a patient for whom reversal was not an option. Linear Gastrointestinal Stapler was used to perform resection of the prolapsed segment and a perfect height stoma reconstructed. We present visual photographs of the steps of the procedure to facilitate learning for our surgical colleagues. Our patient did not require any pain killers or antibiotics and was discharged the next day. Such simple and novel resection techniques for surgical management of prolapsed stoma can easily be learnt by residents and consultants and can provide an important armamentarium in managing this distressing complication.

\section{Introduction}

Stoma prolapse is a common late complication after stoma formation with reported incidence rates of $2-26 \%[1,2]$. The high variability amongst rates can be attributed to variations in anatomical site (ileostomy, transverse, or sigmoid colostomy), stoma formation technique (loop vs end stoma), disease process (benign, malignant, inflammatory) and emergent or elective creation [1]. Although prolapse can materialize with any form of ostomy, it most frequently occurs in the distal limb of a loop colostomy with transverse colostomies possessing the highest risk [3-5]. Prolapsed segments do not carry a life-threatening risk but can potentially induce significant emotional distress in most patients by causing pain and psychological distress [2]. Most patients present due to deterioration in stoma cosmesis and hindrances in fitting stoma appliances. Incarceration is rare and most cases can be managed conservatively with manual reduction. However, in cases where manual reduction is unfavourable due to local edema, local osmotic therapy with topical application of household sugar aids in decreasing the edema and facilitates reduction of the prolapsed segment $[5,6]$. Failure of reduction may induce mucosal irritation with resultant ulceration and bleeding. Surgical intervention may be indicated in rare cases to correct the prolapse to prevent bowel incarceration and strangulation [7]. We herein report a simple technique for local repair with reconstruction of the loop colostomy to avoid laparotomy.

\section{Case Presentation}

A 48 years old gentleman presented to us in clinic with a prolapsed stoma. He was a known case of rectal adenocarcinoma and had undergone a low anterior resection followed by adjuvant chemo radiation in 2015 . He did not have any coexisting comorbidities. In 2016 the patient had developed an extensive fibrosis of the segment of bowel distal to anastomosis and ended up with intestinal obstruction for which a loop transverse colostomy was constructed and adhesiolysis done. For the past 3 months, he had developed a bulge at the stoma site, which was increasing in size, creating skin changes and proving troublesome while fitting the stomal appliance. This was quite distressing to the patient despite taking conservative measures like stoma belt, increasing the size of the stoma appliance and manual reduction of the stoma prolapse. Since reversal of stoma was not an option this patient was planned for revision of stoma with resection technique as described below along with pictorial representation.

\section{Investigation}

The patient was planned for surgery; pre-operative anaesthesia clearance was obtained and relevant preoperative labs done which included Haemoglobin of around $14 \mathrm{~g} / \mathrm{dl}$ with $\mathrm{Cr} 0.1$ and normal electrolytes and a Chest X-ray which was also reported as normal. 


\section{Differential Diagnosis}

After examining our patient in clinic we concluded that he had a prolapsed distal segment of transverse loop colostomy without any parastomal hernia.

\section{Treatment / Management}

The patient was admitted on the day of surgery with nil per oral for six hours. Preoperative antibiotic for prophylaxis was administered prior to induction. General anaesthesia was administered and patient was operated in supine position. After standard prep and drape the stoma was re-examined (Figure 1). Four stay sutures with silk 3/0 were taken in all four quadrants and the prolapsed segment of the transverse colostomy lifted vertically (Figure 2). GIA 100 linear cutting stapler was fired vertically by inserting its jaws into the Stomal lumen first at 3 O'clock position between the stay sutures and the same repeated at 90 'clock (Figure 3 ). The two staple lines bisected the prolapsed segment into two halves (Figure 4). The base of each half was then transacted at approximately $1 \mathrm{~cm}$ above the skin level in perpendicular fashion (Figure 5). On final appearance the circumferential margin of the stoma reconstructed with complete removal of the prolapsed segment and a $1 \mathrm{~cm}$ bud above the skin level was achieved (Figure $6)$.
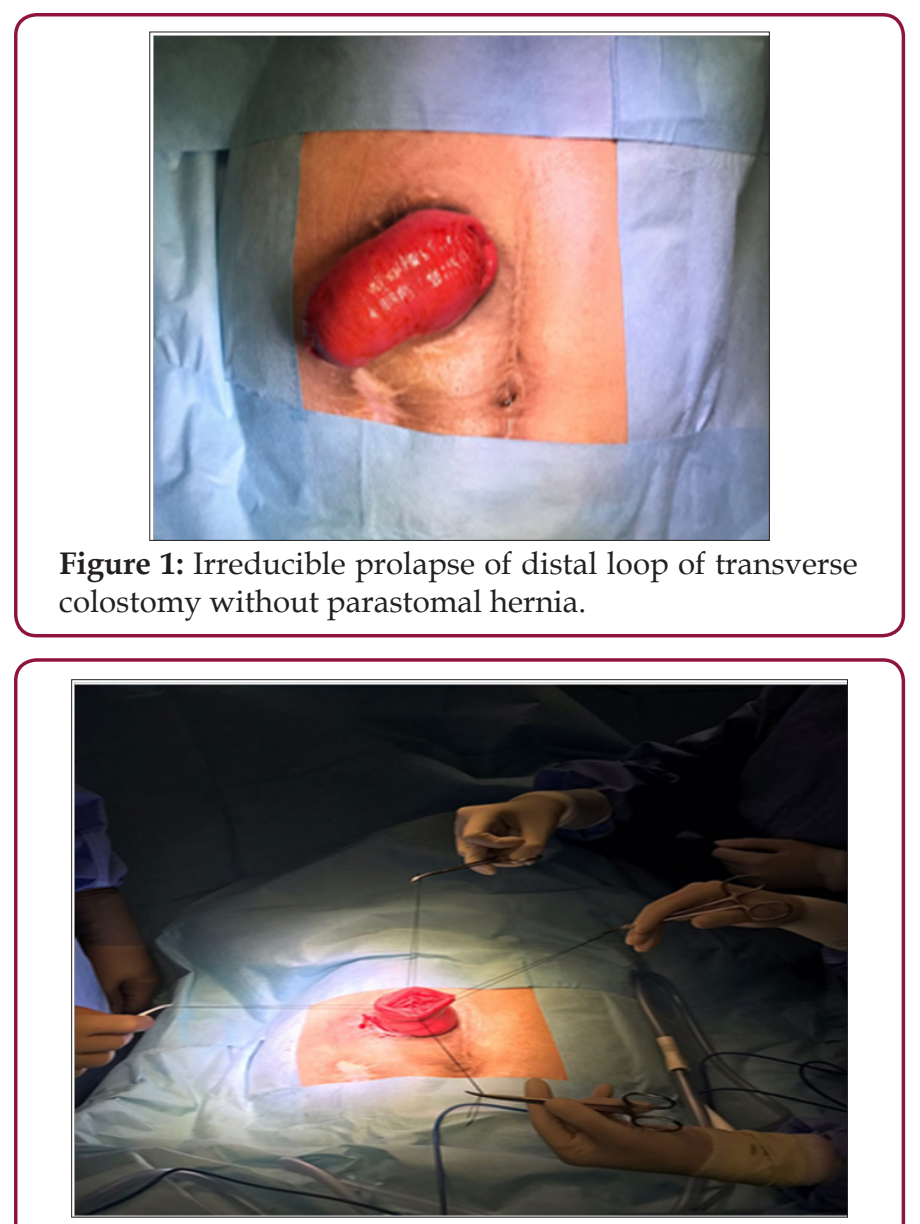

Figure 2: Stay Sutures taken in four quadrants to facilitate lifting the prolapsed segment.

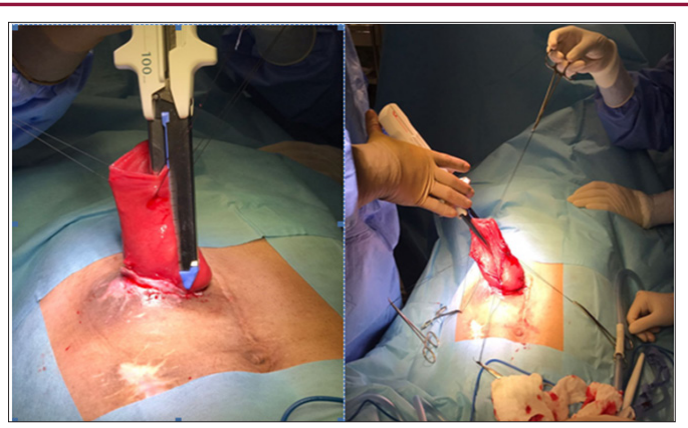

Figure 3: GIA 100 linear staplers was inserted into the lumen of the stoma and used to transect the prolapsed stoma in a longitudinal fashion at $3 \mathrm{O}^{\prime}$ Clock position and then at $9 \mathrm{O}^{\prime}$ Clock.

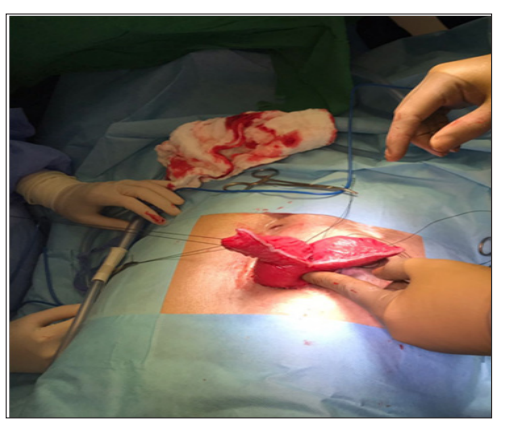

Figure 4: The two staple lines bisected the prolapsed segment into two halves.

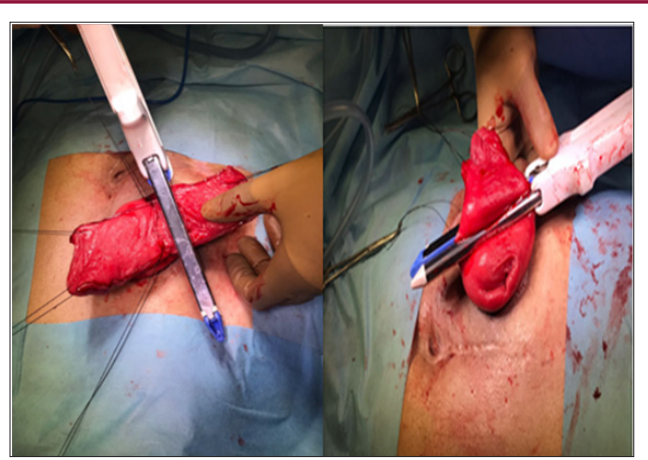

Figure 5: The base of each half was then transected at approximately $1 \mathrm{~cm}$ above the skin level in perpendicular fashion.

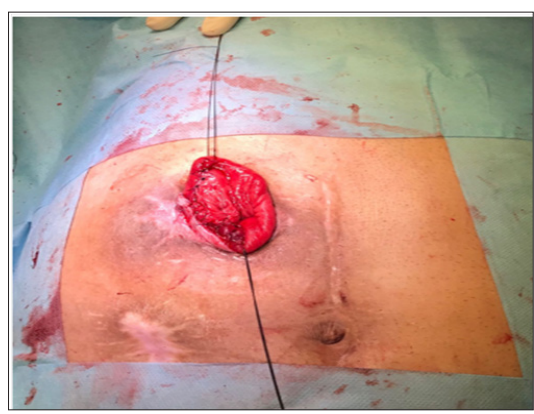

Figure 6: On final appearance the circumferential margin of the stoma reconstructed with complete removal of the prolapsed segment and a $1 \mathrm{~cm}$ bud above the skin level. 


\section{Outcome and Follow-Up}

Our patient did not require any post-operative antibiotics and painkillers. The stoma was well patent and functional and he was discharged the next day without any issues. On follow up in clinic both a week and a month later the stoma was functioning appropriately without any complications.

\section{Discussion}

There are various surgical options for addressing stoma prolapse, with stoma reversal being the best option. However, in some cases reversal may not be a possibility and would require an alternative approach which includes surgical techniques like resection, revision or relocation [8]. Expectant management can sometimes be adopted in cases where timing is the only issue preventing reversal of stoma [9]. During this time conservative measures like belt application, stoma sizing, manual reduction, application of sugar or icing to reduce incarcerated stoma and skin care with application of lubricants like petroleum jelly etc. can be taken. Local resection techniques provide an effective way in dealing with this complication when Stomal prolapse is not associated with parastomal hernia. Sometimes laparotomy with resection of strangulated segment may have to be performed. The complication of stoma prolapse presents late but has a very discomforting and distressful feeling for both patient and the care provider.

Local Resection with linear cutting stapler can provide a unique and effective technique in addressing this issue when the stoma cannot be reversed. The first of this case was reported from Italy in 2003 where instead of linear stapler a circular stapler was used to transect the stoma and the patient did not develop any postoperative recurrence or complications [10]. Similarly, isolated case reports from the United Kingdom, Japan and Greece who adopted the same linear cutting stapler resection techniques resulted in ideal outcomes [11-13]. There is a lack of strong evidence on this technique and future prospective studies need to be undertaken to address this issue. However weak the evidence available, this technique still offers a safe, effective and cost friendly method of managing stoma prolapses.

\section{Learning Points/Take Home Messages}

a. Linear Cutting Stapler resection technique can easily be learnt and reproduced in surgical management of stoma prolapse,

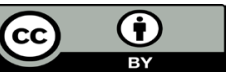

This work is licensed under Creative Commons Attribution 4.0 License

Submission Link: https://biomedres.us/submit-manuscript.php

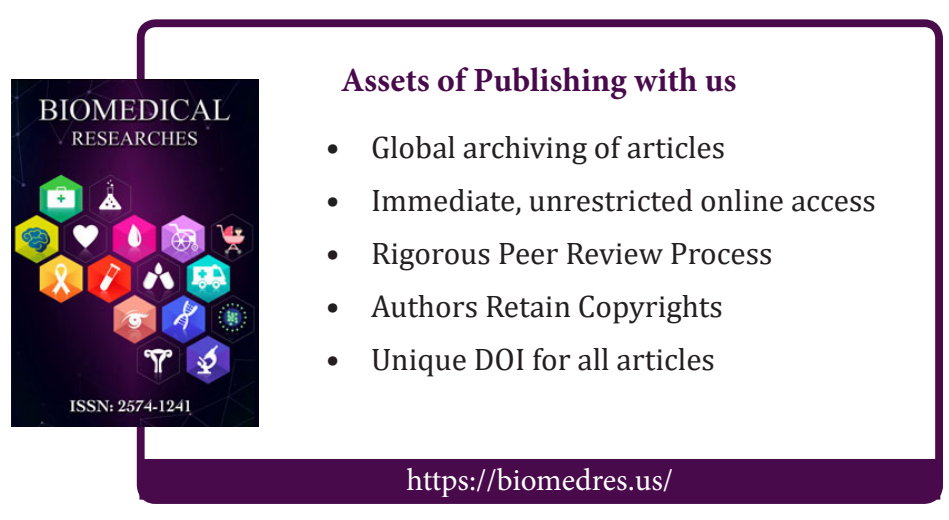

b. Procedure can be reproduced and repeated if recurrence of prolapse occurs,

c. The technique can be performed under local anaesthesia without any need of pain killers and antibiotics,

d. Available literature supports it to be safe and effective.

\section{References}

1. Husain SG, Cataldo TE (2008) Late stomal complications. Clinics in colon and rectal surgery 21(01): 31-40.

2. Monette MM, Harney RT, Morris MS, Chu DI (2016) Local repair of stoma prolapse: Case report of an in vivo application of linear stapler devices. Annals of Medicine and Surgery 11: 32-35.

3. Kim JT, Kumar RR (2006) Reoperation for stoma-related complications. Clinics in colon and rectal surgery 19(04): 207-212.

4. Lees N, Hill J (2003) Intestinal Stomas and their Complications. Surgery (Oxford) 21(2): 39-43.

5. Park JJ, Del Pino A, Orsay CP, Nelson RL, Pearl RK, et al. (1999) Stoma complications. Diseases of the colon \& rectum 42(12): 1575-1580.

6. Shellito PC (1998) Complications of abdominal stoma surgery. Diseases of the colon \& rectum 41(12): 1562-1572.

7. Delrio P, Conzo G (2008) Complications of ileostomy. Seminars in Colon and Rectal Surgery, Elsevier.

8. Bafford AC, Irani JL (2013) Management and complications of stomas. Surgical Clinics 93(1): 145-166.

9. Essani R (2012) Stoma prolapse. Seminars in Colon and Rectal Surgery 23(1): 13-16.

10. Trentin G, De Simone P, Mainente P, Agresta F, Bedin N (2003) Repair of mucosa stomal prolapse with circular stapler. Technical note. Chirurgia italiana 55(1): 141-143.

11. Tepetes K, Spyridakis M, Hatzitheofilou C (2005) Local treatment of a loop colostomy prolapse with a linear stapler. Techniques in coloproctology 9(2): 156-158.

12. Ferguson H, Bhalerao S (2010) Correction of end colostomy prolapse using a curved surgical stapler, performed under sedation. Techniques in coloproctology 14(2): 165-167.

13. Hata F, Kitagawa S, Nishimori H, Furuhata T, Tsuruma T, et al. (2005) A novel, easy, and safe technique to repair a stoma prolapse using a surgical stapling device. Digestive surgery 22(5): 306-310. 